\title{
miR-143 inhibits cell proliferation by targeting autophagy-related 2B in non-small cell lung cancer H1299 cells
}

\author{
JIALI WEI $^{1 *}$, ZHONGLIANG MA ${ }^{1 *}$, YANLI LI ${ }^{1}$, BOTAO ZHAO ${ }^{1}$, DETAO WANG $^{1}$, YAN JIN ${ }^{2}$ and YOUXIN JIN ${ }^{1}$ \\ ${ }^{1}$ School of Life Sciences, Shanghai University, Shanghai 200444; ${ }^{2}$ Institute of Biomedicine and Biotechnology, \\ Shenzhen Institutes of Advanced Technology, Chinese Academy of Sciences, Shenzhen, Guangdong 518055, P.R. China
}

Received January 13, 2014; Accepted August 6, 2014

DOI: $10.3892 / \mathrm{mmr} .2014 .2675$

\begin{abstract}
RNAs (miRNAs) are small, non-coding RNAs involved in multiple biological pathways by regulating post-transcriptional gene expression. Previously, autophagy has been reported to suppress the progression of non-small cell lung cancer (NSCLC). However, how miRNAs regulate autophagy in NSCLC remains to be elucidated. In the present study, the autophagy gene, autophagy-related 2B (ATG2B), was identified as a novel target of miR-143. The overexpression of miR-143 was able to downregulate the expression of $\operatorname{atg} 2 b$ at the transcriptional and translational levels by direct binding to its $3^{\prime}$ untranslated region. Cell proliferation was significantly inhibited by the ectopic expression of miR-143 in H1299 cells. Knockdown of ATG2B resulted in a similar phenotype, with the overexpression of miR-143 in NSCLC cells. Furthermore, knockdown of ATG2B and hexokinase 2, a key enzyme in glycolysis and another target of miR-143, co-ordinated to inhibit the proliferation of H1299 cells. The results of the present study demonstrated that miR-143 was a novel and important regulator of autophagy by targeting $A T G 2 B$ and repression of gene expression in autophagy and high glycolysis had a coordinate effect in H1299 cells. These results suggested that ATG2B may be a new potential therapeutic target for NSCLC. Furthermore, it was implied that interrupting autophagy and glycolysis improves NSCLC therapy.
\end{abstract}

Correspondence to: Professor Youxin Jin, School of Life Sciences, Shanghai University, 99 Shangda Road, Shanghai 200444, P.R. China

E-mail: jinyouxin@shu.edu.cn

Dr Yan Jin, Institute of Biomedicine and Biotechnology, Shenzhen Institutes of Advanced Technology, Chinese Academy of Sciences, 1068 Xueyuan Boulevard, Shenzhen, Guangdong 518055, P.R. China E-mail: yan.jin@siat.ac.cn

*Contributed equally

Key words: microRNA-143, autophagy-related 2B, microRNA, non-small cell lung cancer, autophagy, glycolysis

\section{Introduction}

Autophagy is a regulated intracellular degradation process in lysosomes for recycling damaged organelles and toxic proteins to maintain cell homeostasis (1). Under cellular stresses, including nutrient deprivation, the promotion of autophagy protects cells from pathways of cell death (2-5). Autophagy-deficient mice died within 1 day following birth due to a shortage of nutrients (6). However, this survival mechanism also assists cancer cells in overcoming starvation and hypoxia. Several studies have demonstrated that autophagy is involved in the progression of cancer (7-9). In the past decade, studies focusing on autophagy have advanced following the identification of a large number of autophagy-related genes in yeast, a significant proportion of which are conserved in humans $(10,11)$. However, the regulation of autophagy is complex and the molecular mechanisms in humans, particularly in cancer cells, remain to be elucidated. The function of autophagy-related 2 (ATG2) is not well characterized in humans and only one study has demonstrated that the homologous ATG2A and ATG2B genes are involved in autophagosome formation in HeLa cells (12). It has previously been reported that microRNA (miR)-130a is able to target $A T G 2 B$ to inhibit autophagic flux and induce cell death in human chronic lymphocytic leukemia (CLL) (5).

microRNAs (miRNAs) are a group of small, non-coding RNAs. They are important in diverse biological processes, including development, differentiation, the cell cycle and apoptosis $(13,14)$, through post-transcriptional gene regulation. Previous studies have demonstrated that miRNAs are involved in the regulation of autophagy $(15,16)$. At present, $\sim 20$ miRNAs have been reported to target ATG-related genes $(15,16)$, among which include the core components of autophagy (15). However, the post-transcriptional regulation of autophagy by miRNAs remains to be fully elucidated. It is necessary to investigate the complicated regulatory mechanisms of autophagy by miRNAs, which may have the potential to be developed in cancer therapy.

Lung cancer is the leading cause of cancer mortality worldwide and non-small cell lung cancer (NSCLC) accounts for $\sim 80 \%$ of lung cancer. The five-year survival rate of NSCLC is only $15 \%$ and the majority of patients with NSCLC succumb to this disease due to ineffective therapy and poor diagnostic tools during its early stages (17). It has been reported that 
miR-143 acts as a tumor suppressor by inhibiting cell proliferation in NSCLC (18).

\section{Materials and methods}

Cell culture. All cell lines were cultured at $37^{\circ} \mathrm{C}$ in a humidified atmosphere containing $5 \% \mathrm{CO}_{2}$. The NSCLC cell lines A549, H1299, Spca-1, HCC827 and Beas-2B were obtained from the American Type Culture Collection (Manassas, VA, USA) and cultured according to the manufacturer's instructions. H23 and 95-D were purchased from the Cell Bank of the Chinese Academy of Sciences (Shanghai, China). H23 and 95-D were maintained in RPMI-1640 medium with $10 \%$ fetal bovine serum (FBS). The medium and FBS of all cell lines were purchased from Invitrogen Life Technologies (Gaithersburg, MD, USA). Lipofectamine 2000 (Invitrogen Life Technologies) was used for cell transfection according to the manufacturer's instructions.

A549, HCC827, Spca-1, 95-D, HEK293T and Beas-2B cells were obtained from the Cell Bank of the Chinese Academy of Sciences. H1299 and H23 cells were purchased from the American Type Culture Collection. A549, HCC827, H1299, H23 and Spca-1 cell lines belong to the NSCLC cell line. A549 cells were cultured in F-12K medium (Gibco-BRL, Gaithersburg, MD, USA). HCC827, H1299 and Spca-1 cells were cultured in Dulbecco's modified Eagle's medium (DMEM; Gibco-BRL) with the exception of the A549 cells. H23 and 95-D were maintained in RPMI-1640 medium. The Beas-2B cell line was isolated from normal human bronchial epithelium. Beas-2B cells were cultured in LHC-9 medium, including epidermal growth factor (EGF), hydrocortisone and adrenaline. All media were supplemented with $10 \% \mathrm{FBS}$, $100 \mathrm{U} / \mathrm{ml}$ penicillin and $100 \mu \mathrm{g} / \mathrm{ml}$ streptomycin (Sangon Biotech, Shanghai, China). Lipofectamine 2000 (Invitrogen Life Technologies) was used for cell transfection according to the manufacturer's instructions.

RNA extraction and reverse transcription quantitative polymerase chain reaction ( $R T-q P C R)$. Total RNA for RT-qPCR was extracted using TRIzol (Sangon Biotech, Shanghai, China) according to the manufacturer's instructions. For detection of miR-143, reverse transcription was performed using a One step PrimeScript ${ }^{\circledR}$ miRNA cDNA Synthesis kit (Takara Bio, Inc., Shiga, Japan). For analysis of coding gene expression, the cDNA was synthesized by reverse transcription using a PrimeScript $^{\mathrm{TM}}$ 1st Strand cDNA Synthesis kit (Takara Bio, Inc.). RT-qPCR was performed using SYBR Green Reagents (Bio-Rad, Hercules, CA, USA) on the iQ5 Real-Time PCR Detection system (Bio-Rad). Expression of ATG2B and HK2 relative to $18 \mathrm{~S}$ and $\mathrm{miR}-143$ relative to $\mathrm{U} 6$ was calculated using the $2^{-\Delta \Delta \mathrm{Ct}}$ method. miR-143 was amplified using the forward primer 5'-TGAGATGAAGCACTGTAGCTC-3' and the reverse Uni-miR RT-qPCR primer (Takara Bio, Inc.). The ATG2B and HK2 primers for RT-qPCR were used according to the previous design $(5,18)$. 18S RNA was detected using the following primers: forward 5'-TGAGATGAAGCACTGTAGCTC-3' and reverse 5'-TAGTAGCGACGGGCGGTGTG-3'. The RT-qPCR primers for U6 were as follows: forward 5'-CTCGCTTCGGCAGCACA-3' and reverse 5'-AACGCTTCACGAATTTGCGT-3'.
Western blot analysis. Proteins were extracted from cells using radioimmunoprecipitation assay lysis buffer (Beyotime Institute of Biotechnology, Shanghai, China) and separated using sodium dodecyl sulfate polyacrylamide gel electrophoresis. Subsequently, proteins were transferred from the gel onto a polyvinylidene difluoride membrane Merck Millipore $\square$ Billerica, MA, USA. The primary antibodies used in the assay were rabbit anti-ATG2B (1:1000) and rabbit anti-GAPDH (1:1000). The secondary antibodies were goatanti-rabbit (1:10000). Following incubation with the specific antibody overnight followed by washing and incubating with a secondary antibody, protein expression was detected using a Pierce $^{\mathrm{TM}}$ ECL Western Blotting substrate (Thermo Fisher Scientific, Inc., Rockford, IL, USA) and images were captured using a ChemiDoc XRS (Bio-Rad). GAPDH rabbit antibody was purchased from Cell Signaling Technology, Inc. (Danvers, MA, USA) and ATG2B antibody produced in rabbit was purchased from Abcam (Cambridge, UK).

Luciferase reporter assay. The 3' untranslated region (3'UTR) of ATG2B was amplified using the following primers: forward 5'-CGGAATTCTGGCTTGGAACTGACAGTGT-3' and reverse 5'-AAACTGCAGCACATTCCTAACAAGCCTGC-3'. The DNA fragment was ligated into the pGL3 vector (Promega $\mathrm{GmbH}$, Mannheim, Germany) using two restriction enzymes, EcoRI and PstI. A mutant 3'UTR of ATG2B was generated using a QuikChange ${ }^{\circledR}$ Site-Directed Mutagenesis kit (Stratagene, La Jolla, CA, USA). Each reporter construct was co-transfected into $293 \mathrm{~T}$ cells in 24-well plates with the thymidine kinase Renilla luciferase plasmid (pRL-TK) for use in a Dual-Luciferase reporter assay, together with miR-143 mimic or negative control RNA (Riobo, Guangzhou, China). The cells were harvested $48 \mathrm{~h}$ after transfection and the luciferase activity was measured using a Dual-Luciferase reporter assay system (Promega, Madison, WI, USA) and normalized to the pRL-TK control.

Cell proliferation assay. Cells were seeded into 96-well plates at 2,000 cells/well after $6 \mathrm{~h}$ of transfection. The Cell Counting kit-8 (CCK-8; Dojindo Laboratories, Kumamoto, Japan) was used to detect the relative cell proliferation for 4 days. Prior to detection, $5 \mu \mathrm{l}$ CCK-8 agent was added to the media for $3 \mathrm{~h}$ at $37^{\circ} \mathrm{C}$ and light absorbance was then measured at $450 \mathrm{~nm}$. The siRNA used for knockdown of HK2 and ATG2B was performed as previously described $(5,18)$.

Statistical analysis. Student's t-test was performed to compare the data of two experimental groups. $\mathrm{P}<0.05$ was considered to indicate a statistically significant difference.

\section{Results}

Target prediction and functional analysis of miR-143. Previous studies have demonstrated that miR-143 is important in various types of cancer via different biological pathways (19-22). The diverse functions of miR-143 have been observed in various tumor systems, and prompted the investigation of the complex regulatory mechanisms of miR-143 (18-26,30). To determine the downstream signaling of miR-143, four miRNA target prediction programs 
(miRDB, TargetScan5.2, DIANA-microT3.0 and miRanda) were used to predict its putative targets. As results were obtained using two or more independent programs, a total of 28 more promising targets were selected from the hundreds of putative targets of miR-143 (Table I). These genes were further categorized into the various pathways and biological processes involved in tumorigenesis using the University of California Santa Cruz Genome browser (genome.ucsc.edu), including CREBZF in the transforming growth factor- $\beta$ signaling pathway, cysteine-rich with EGF-like domain protein 1 and Kruppel-like factor 5 in the EGF signaling pathway, insulin-like growth factor-binding protein-5 in the insulin-like growth factor signaling pathway, cyclin-dependent kinase 1 in the cell cycle and ATG2B in autophagy regulation.

$A T G 2 B$, a novel target of $m i R-143$. ATG2B, an autophagy related gene, was identified due to its prediction by three single prediction programs (Table I). The seeding region of miR-143 was located in the 3'UTR of ATG2B (TargetScan5.2), which is conserved across species, including human, chimpanzee, rhesus, mouse, rat, guinea pig and rabbit (Fig. 1A). To verify whether miR-143 directly regulated ATG2B by binding to its 3'UTR, the full-length 3'UTR of ATG2B was constructed into the downstream of the firefly luciferase gene in the luciferase reporter vector PGL3 (luci-ATG2B-3'UTR). In addition, mutation of the conservative seeding region of ATG2B 3'UTR (luci-ATG2B-3'UTR ${ }^{\text {mut }}$ ) was constructed as a negative control (Fig. 1B). A luciferase assay was performed in the 293 T cells. When co-transfected with luci-ATG2B-3'UTR, the miR-143 mimics reduced the activity of luciferase to $\sim 70 \%$ compared with the mimic negative control (Fig. 1C). Notably, this significant inhibition by miR-143 was eliminated on the conservative seeding region of the mutated plasmid luci-ATG2B-3'UTR ${ }^{\text {mut }}$, (Fig. 1C). Taken together, these results demonstrated that miR-143 directly binds to the conservative seed sequence of ATG2B 3'UTR to negatively regulate the expression of $\mathrm{ATG} 2 \mathrm{~B}$.

Negative regulation of $A T G 2 B$ by $m i R-143$ in NSCLC. It is well established that autophagy is involved in the development of cancer (7). ATG2B, the miR-143 target verified in the present study, is a key component involved in autophagosome formation (12). Inhibition of ATG2B-related autophagic flux induces cell death in CLL (5). However, the contribution of autophagy in NSCLC, particularly in autophagy regulated by miRNA in NSCLC, remains to be elucidated. In the present study, miR-143/ATG2B was evaluated in NSCLCs. The results from the RT-qPCR of miR-143 in NSCLC cell lines H1299, A549, spca-1 and 95-D (Fig. 2B) were consistent with previous studies that miR-143 was downregulated in NSCLC cell lines. Notably, the ATG2B mRNA was significantly upregulated in all six NSCLC cell lines compared with the normal lung BEAS-2B cells (Fig. 2A), which demonstrated reverse correlation with the expression of miR-143.

To determine whether the upregulation of ATG2B results from the loss of miR-143 in NSCLCs, the level of ATG2B was monitored in differing levels of miR-143 expression. The upregulated expression of ATG2B in H1299 cells was restored by transfection with miR-143 mimics, while upregu-
Table I. List of potential target genes of hsa-143.

\begin{tabular}{|c|c|c|c|c|}
\hline Gene name & miRDB & $\begin{array}{c}\text { Target } \\
\text { Scan5.2 }\end{array}$ & $\begin{array}{l}\text { DIANA- } \\
\text { microT3.0 }\end{array}$ & miRanda \\
\hline MAP3K7 & $\sqrt{ }$ & $\sqrt{ }$ & & $\sqrt{ }$ \\
\hline CREB1 & & & $\sqrt{ }$ & $\sqrt{ }$ \\
\hline KLF5 & $\sqrt{ }$ & $\sqrt{ }$ & & $\sqrt{ }$ \\
\hline MAPK7 & $\sqrt{ }$ & $\sqrt{ }$ & $\sqrt{ }$ & $\sqrt{ }$ \\
\hline MYO6 & & $\sqrt{ }$ & $\sqrt{ }$ & $\sqrt{ }$ \\
\hline ABL2 & $\sqrt{ }$ & $\sqrt{ }$ & $\sqrt{ }$ & \\
\hline ETV6 & $\sqrt{ }$ & $\sqrt{ }$ & & $\sqrt{ }$ \\
\hline IGFBP5 & $\sqrt{ }$ & $\sqrt{ }$ & $\sqrt{ }$ & $\sqrt{ }$ \\
\hline SIX4 & $\sqrt{ }$ & $\sqrt{ }$ & & $\sqrt{ }$ \\
\hline WDR7 & $\sqrt{ }$ & & $\sqrt{ }$ & \\
\hline SIRT5 & $\sqrt{ }$ & & & $\sqrt{ }$ \\
\hline ITGA6 & $\sqrt{ }$ & $\sqrt{ }$ & & \\
\hline TUB & $\sqrt{ }$ & $\sqrt{ }$ & $\sqrt{ }$ & $\sqrt{ }$ \\
\hline KLF5 & $\sqrt{ }$ & $\sqrt{ }$ & & $\sqrt{ }$ \\
\hline CDK1 & $\sqrt{ }$ & & & $\sqrt{ }$ \\
\hline TET1 & $\sqrt{ }$ & $\sqrt{ }$ & & $\sqrt{ }$ \\
\hline STAC & $\sqrt{ }$ & $\sqrt{ }$ & & \\
\hline CREBZF & $\sqrt{ }$ & $\sqrt{ }$ & & $\sqrt{ }$ \\
\hline VAPB & $\sqrt{ }$ & $\sqrt{ }$ & $\sqrt{ }$ & \\
\hline $\mathrm{PC}$ & $\sqrt{ }$ & $\sqrt{ }$ & & $\sqrt{ }$ \\
\hline ETV6 & $\sqrt{ }$ & $\sqrt{ }$ & & $\sqrt{ }$ \\
\hline ITGB8 & $\sqrt{ }$ & & & $\sqrt{ }$ \\
\hline ATG2B & $\sqrt{ }$ & $\sqrt{ }$ & $\sqrt{ }$ & \\
\hline CRELD1 & $\sqrt{ }$ & $\sqrt{ }$ & $\sqrt{ }$ & \\
\hline ARHGAP26 & & $\sqrt{ }$ & $\sqrt{ }$ & \\
\hline MAF & $\sqrt{ }$ & & & $\sqrt{ }$ \\
\hline SSH2 & & $\sqrt{ }$ & $\sqrt{ }$ & \\
\hline MY09A & & & $\sqrt{ }$ & $\sqrt{ }$ \\
\hline
\end{tabular}

lation of ATG2B was identified in cells transfected with the miR-143 inhibitor (Fig. 1D and E). These data indicated that miR-143 negatively regulated ATG2B in the NSCLC cells and suppression by miR-143 decreased the level of ATG2B mRNA (Fig. 1D).

Growth inhibition of NSCLC cells by miR-143 via co-ordinate silencing of $A T G 2 B$ and $H K 2$. To investigate the contribution of ATG2B, the novel target of miR-143, to growth inhibition, the expression of ATG2B was knocked down by siRNA-mediated silencing. (Fig. 3A and C). The results demonstrated that the proliferation of $\mathrm{H} 1299$ cells transfected with siATG2B decreased by $\sim 10 \%$ compared with the cells transfected with the negative control siRNA (Fig. 3D), which suggested that miR-143 inhibited NSCLC cell proliferation, at least partially, via its negative regulation of ATG2B.

A previous study demonstrated that miR-143 targets HK2, a key enzyme in the high glycolysis (Warburg effect) of a tumor, to inhibit the proliferation of the NSCLC cell line H1299 (18). The results of the present study also demonstrated that the mRNA expression of HK2 was mark- 


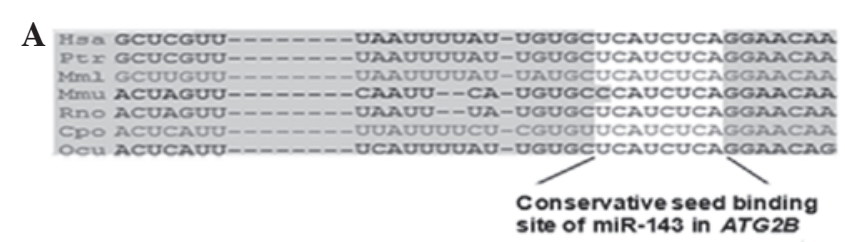

B

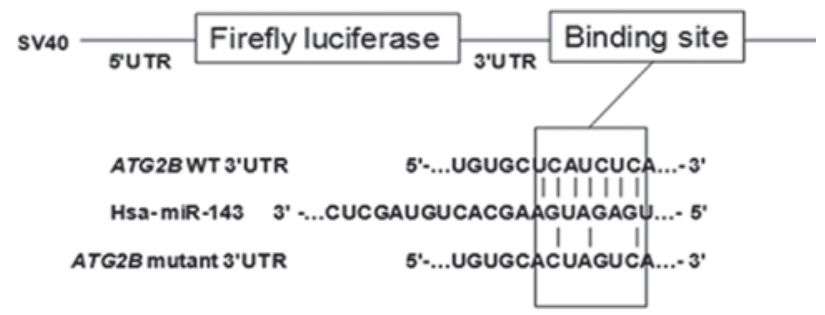

C

ATG2B 3'UTR+NC

四 ATG2B 3'UTR+miR-143

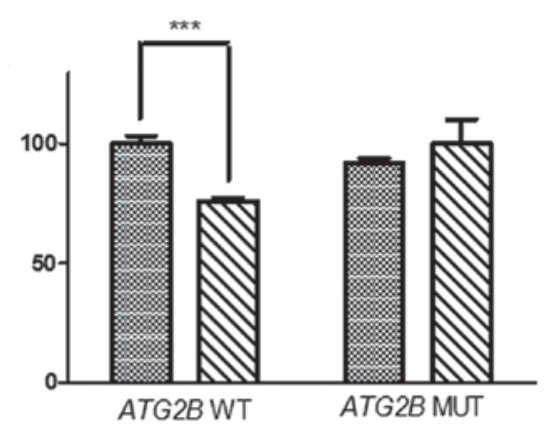

D

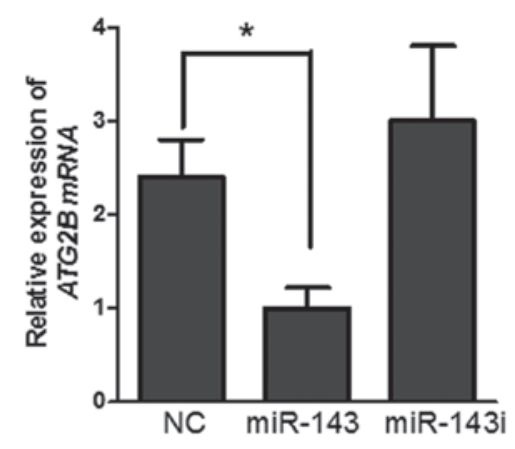

$\mathbf{E}$

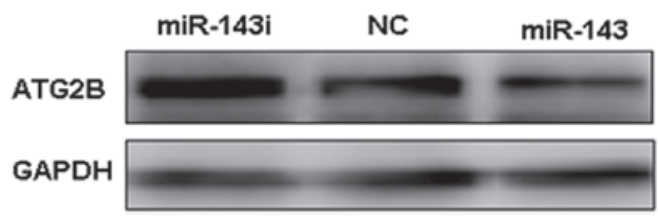

Figure 1. ATG2B is a direct target of miR-143. (A) Conservative seed binding sites of miR-143 in $A T G 2 B$ in different species. (B) Schematic representation of the $3^{\prime} \mathrm{UTR}$ of $A T G 2 B$ constructed downstream of the firefly luciferase gene in the reporter vector pGL3. (C) Luciferase reporter assay using wild type binding site of $A T G 2 B 3^{\prime} \mathrm{UTR}$ ( $A T G 2 B$ WT) and mutated binding site of $A T G 2 B 3^{\prime} \mathrm{UTR}$ (ATG2B MUT) ${ }^{* * *} \mathrm{P}<0.001$ compared with ATG2B 3'UTR + miR-143 mimics. (D) Relative mRNA expression of ATG2B in H1299 cells transfected with NC, miR-143 mimics and miR-143 inhibitor was determined by reverse transcription quantitative polymerase chain reaction. " $\mathrm{P}<0.05$ compared with the gene expression in the cell treated with $\mathrm{NC}$ and miR-143 mimics. (E) Protein expression level of ATG2B in H1299 cells transfected with NC, miR-143 mimics and miR-143 inhibitor was determined by western blot analysis. ATG2B, autophagy-related 2B; miR, microRNA; 3'UTR, 3' untranslated region; ATG2b WT, wild type binding site of ATG2B 3'UTR; ATG2B MUT, mutated binding site of ATG2B 3'UTR; NC, negative control.
A

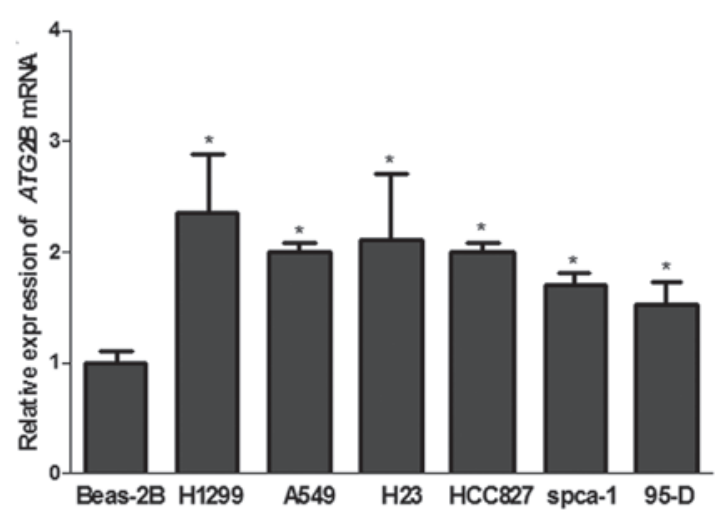

B

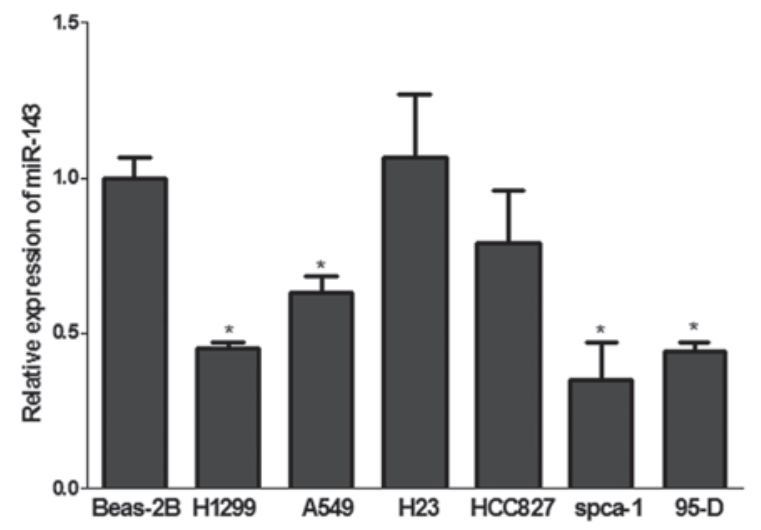

Figure 2. Relative expression of ATG2B and miR-143 in different NSCLC cell lines. (A) Expression of ATG2B in NSCLC cell lines detected by RT-qPCR. (B) Expression of miR-143 in NSCLC cell lines detected by RT-qPCR. " $\mathrm{P}<0.05$ compared with the normal lung cell line Beas-2B. ATG2B, autophagy-related 2B; miR, microRNA; NSCLC, non small cell lung cancer; RT-qPCR, reverse transcription quantitative polymerase chain reaction.

edly decreased and the cell proliferation was significantly inhibited in the H1299 cells transfected with HK2 siRNA (siHK2) compared with those transfected with negative control siRNA (Fig. 3B). Furthermore, the inhibition of cell proliferation was more significant in the H1299 cells co-transfected with siATG2B and siHK2 compared with those transfected with either siATG2B or siHK2 alone, which was similar to the potent inhibitory effect of miR-143 (Fig. 3D). From these data, it is possible to conclude that miR-143 targeted ATG2B and HK2 to suppress the growth of NSCLC cells more efficiently. Interruption of autophagy and the Warburg effect provides a potentially efficient therapeutic strategy against NSCLC.

\section{Discussion}

Emerging studies have demonstrated the effects of miR-143 in various types of human cancer $(18-26,30)$. miR-143 functions as a tumor suppressor through targeting different oncogenes in various pathways (Fig. 4) in pancreatic cancer (21), bladder cancer (23), colorectal cancer (20), breast cancer (24), esophageal squamous cell carcinoma (25), osteosarcoma (26) and lung cancer $(18,19)$. In NSCLC, the loss of miR-143 promotes tumor proliferation, migration and invasion through targeting the Kras, CD44v3 and HK2 genes $(18,19)$. For the first time, to the best of our knowledge, the present study linked miR-143 to the autophagy pathway. 

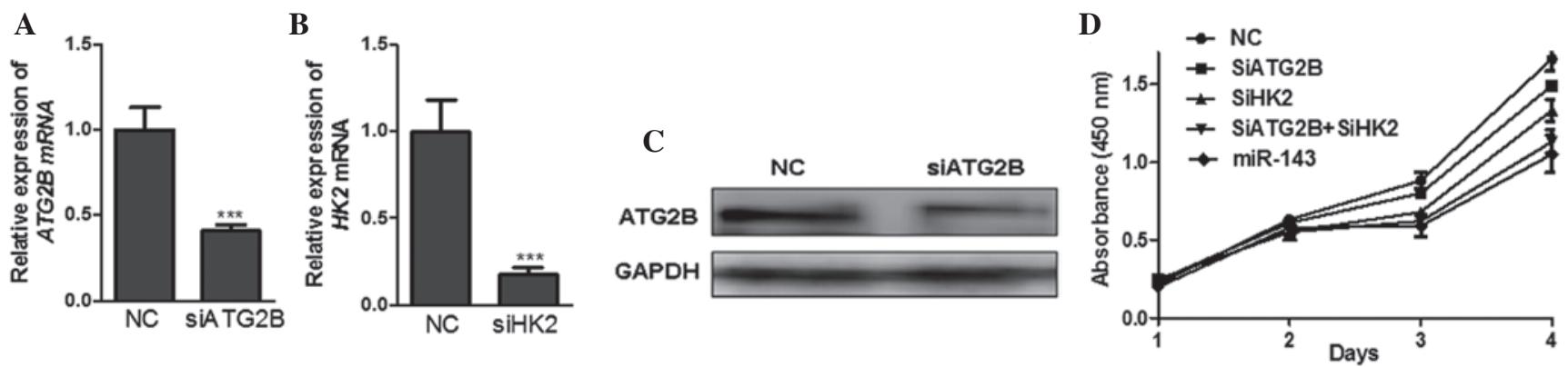

Figure 3. Knock down of ATG2B and HK2 inhibited the proliferation of H1299 cells. (A and B) mRNA level of ATG2B and $H K 2$ in H1299 cells transfected with siATG2B or siHK2-knockdown. ${ }^{* * *} \mathrm{P}<0.001$. (C) Protein expression level of ATG2B in H1299 cells transfected with siATG2B. (D) Cell proliferation was determined for 4 days using a Cell Counting kit-8 assay in H1299 cells transfected with NC, miR-143, siATG2B, siHK2 and a mixture of siATG2B and siHK2. ATG2B, autophagy-related 2B; miR, microRNA; HK2, hexokinase 2; HK2si, HK siRNA NC, negative control.

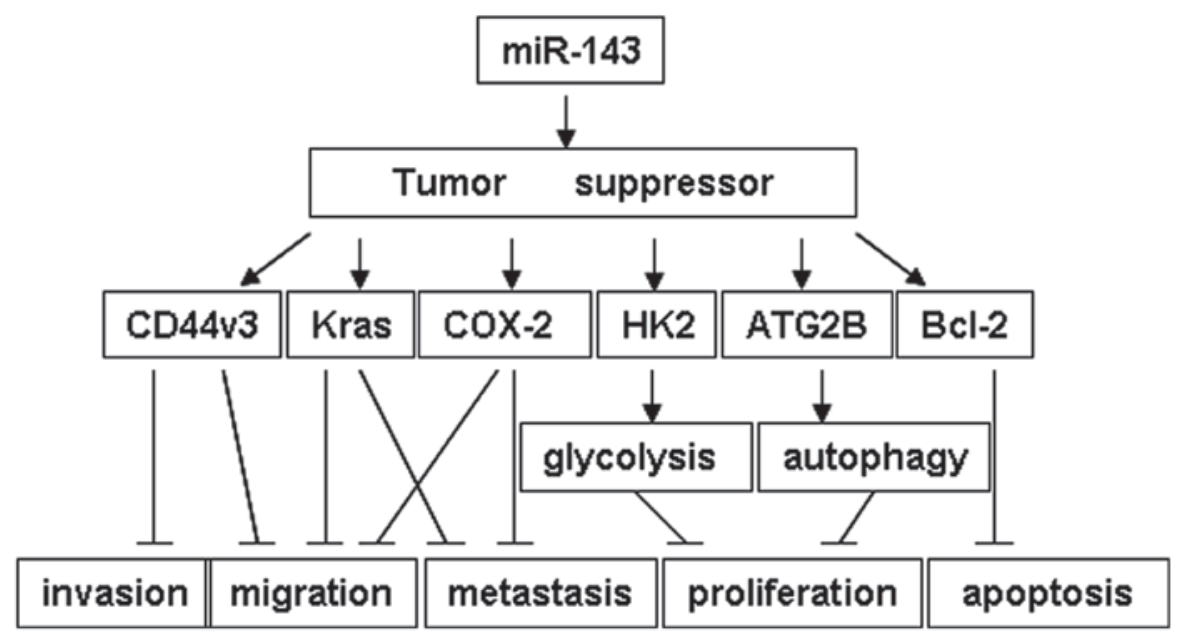

Figure 4. miR-143 acts as a tumor suppressor via its target genes in various processes. miR, microRNA; COX-2, cyclooxygenase 2; HK2, hexokinase 2; Bcl-2, B-cell lymphoma 2; ATG2B, autophagy-related 2B; Kras, Kirsten rat sarcoma 2.

$A T G 2 B$, an autophagy related gene, was demonstrated to be a target of miR-143 (Fig. 4).

There have been several studies suggesting that autophagy is important in NSCLC. A549 cells present higher sensitivity to the chemotherapeutic chemical 5-fluorouracil when the autophagic response is attenuated by knockdown of the ATG-related gene ATG7 (27). In the mouse model $\left(\operatorname{atg} 7^{-/}\right.$), an autophagic defect was demonstrated to suppress the progression of K-ras-induced lung tumor (8). In addition, miR-130a inhibits the autophagic flux and induces cell death in human CLL via direct targeting to ATG2B (5), which involves the formation of an autophagosome (12). The present study also demonstrated that miR-143 was able to downregulate the expression of ATG2B and suppress cell proliferation in H1299 cells. To the best of our knowledge, this is the first study investigating miR-related autophagy regulation in NSCLC. It is known that autophagy is not only important in maintaining cell homeostasis by degrading damaged organelles and proteotoxic waste, but it also functions in stress and starvation conditions (2-5). The upregulation of $A T G 2 B$ mRNA in lung cancer patients and NSCLC cell lines indicated that the progression of cancer requires autophagy for survival more urgently than in normal cells.
The Warburg effect is the process of high glycolysis, which occurs in the majority of cancer cells, converting glucose into lactic acid even in the presence of oxygen (28). This alternative metabolic process enables tumor cells to obtain more efficient energy for survival. HK2 is a key enzyme in glycolysis $(22,29)$. Studies have demonstrated that miR-143 targets HK2 to inhibit the Warburg effect, resulting in the inhibition of cell proliferation in colon cancer (22), breast cancer (24), renal cell cancer (30) and NSCLC (18). In the present study, the result of HK2 knockdown by siRNA-mediated silencing in the H1299 cells was consistent with previous studies (18). Furthermore, the results revealed that cell survival was reduced more significantly following HK2 and ATG2B knockdown by RNAi in the H1299 cells. Therefore, it can be deduced that when cancer cells lack energy, the function of autophagy becomes essential. The possible cross-talk between HK2 and ATG2B or between the Warburg effect and autophagy requires investigation in future studies.

\section{Acknowledgements}

This study was supported by grants from the National Basic Research Program of China (no. 2011CBA01105), the National 
Natural Science Foundation of China (nos. 31170750 and 31100570), the Innovation Program of Shanghai Municipal Commission of the Sciences and Technology (no. 11ZR141220) and from the State Key Laboratory of Molecular Biology, Institute of Biochemistry and Molecular Biology, Shanghai Institutes of Life Sciences, Chinese Academy of Sciences.

\section{References}

1. He C and Klionsky DJ: Regulation mechanisms and signaling pathways of autophagy. Annu Rev Genet 43: 67-93, 2009.

2. Kroemer G, Mariño G and Levine B: Autophagy and the integrated stress response. Mol Cell 40: 280-293, 2010.

3. Mathew R, Karantza-Wadsworth V and White E: Role of autophagy in cancer. Nat Rev Cancer 7: 961-967, 2007.

4. Altman BJ and Rathmell JC: Metabolic stress in autophagy and cell death pathways. Cold Spring Harb Perspect Biol 4: a008763, 2012.

5. Kovaleva V, Mora R, Park YJ, et al: miRNA-130a targets ATG2B and DICER 1 to inhibit autophagy and trigger killing of chronic lymphocytic leukemia cells. Cancer Res 72: 1763-1772, 2012.

6. Kuma A, Hatano M, Matsui M, et al: The role of autophagy during the early neonatal starvation period. Nature 432: 1032-1036, 2004.

7. Shintani T and Klionsky DJ: Autophagy in health and disease: a double-edged sword. Science 306: 990-995, 2004.

8. Guo JY, Karsli-Uzunbas G, Mathew R, et al: Autophagy suppresses progression of K-ras-induced lung tumors to oncocytomas and maintains lipid homeostasis. Genes Dev 27: 1447-1461, 2013.

9. Kondo Y, Kanzawa T, Sawaya R and Kondo S: The role of autophagy in cancer development and response to therapy. Nat Rev Cancer 5: 726-734, 2005.

10. Xie Z and Klionsky DJ: Autophagosome formation: core machinery and adaptations. Nat Cell Biol 9: 1102-1109, 2007.

11. Yang Z and Klionsky DJ: Mammalian autophagy: core molecular machinery and signaling regulation. Curr Opin Cell Biol 22: 124-131, 2010.

12. Velikkakath AK, Nishimura T, OitaE, Ishihara N and Mizushima N: Mammalian Atg2 proteins are essential for autophagosome formation and important for regulation of size and distribution of lipid droplets. Mol Biol Cell 23: 896-909, 2012.

13. Flynt AS andLaiEC: Biological principles of microRNA-mediated regulation: shared themes amid diversity. Nat Rev Genet 9: 831-842, 2008.

14. Ventura A and Jacks T: MicroRNAs and cancer: short RNAs go a long way. Cell 136: 586-591, 2009.

15. Frankel LB and Lund AH: MicroRNA regulation of autophagy. Carcinogenesis 33: 2018-2025, 2012.
16. Zhai H, Fesler A and Ju J: MicroRNA: a third dimension in autophagy. Cell Cycle 12: 246-250, 2013.

17. Molina JR, Yang P, Cassivi SD, Schild SE and Adjei AA: Non-small cell lung cancer: epidemiology, risk factors, treatment, and survivorship. Mayo Clin Proc 83: 584-594, 2008

18. Fang R, Xiao T, Fang Z, et al: MicroRNA-143 (miR-143) regulates cancer glycolysis via targeting hexokinase 2 gene. J Biol Chem 287: 23227-23235, 2012.

19. Ma Q, Jiang Q, Pu Q, et al: MicroRNA-143 inhibits migration and invasion of human non-small-cell lung cancer and its relative mechanism. Int J Biol Sci 9: 680-692, 2013.

20. Qian X, Yu J, Yin Y, et al: MicroRNA-143 inhibits tumor growth and angiogenesis and sensitizes chemosensitivity to oxaliplatin in colorectal cancers. Cell Cycle 12: 1385-1394, 2013.

21. Hu Y, Ou Y, Wu K, Chen Y and Sun W: miR-143 inhibits the metastasis of pancreatic cancer and an associated signaling pathway. Tumor Biology 33: 1863-1870, 2012.

22. Gregersen LH, Jacobsen A, Frankel LB, Wen J, Krogh A and Lund AH: MicroRNA-143 down-regulates Hexokinase 2 in colon cancer cells. BMC Cancer 12: 232, 2012.

23. Song T, Zhang X, Wang C, et al: Expression of miR-143 reduces growth and migration of human bladder carcinoma cells by targeting cyclooxygenase-2. Asian Pac J Cancer Prev 12: 929-933, 2011.

24. Jiang S, Zhang LF, Zhang HW, et al: A novel miR-155/miR-143 cascade controls glycolysis by regulating hexokinase 2 in breast cancer cells. EMBO J 31: 1985-1998, 2012.

25. Ni Y, Meng L, Wang L, et al: MicroRNA-143 functions as a tumor suppressor in human esophageal squamous cell carcinoma. Gene 517: 197-204, 2013.

26. Zhang H, Cai X, Wang Y, Tang H, Tong D and Ji F: microRNA-143, down-regulated in osteosarcoma, promotes apoptosis and suppresses tumorigenicity by targeting Bcl-2. Oncol Rep 24: 1363-1369, 2010.

27. Pan X, Zhang X, Sun H, Zhang J, Yan M and Zhang H: Autophagy inhibition promotes 5 -fluorouraci-induced apoptosis by stimulating ROS formation in human non-small cell lung cancer A549 cells. PLoS One 8: e56679, 2013

28. Warburg O: On the origin of cancer cells. Science 123: 309-314, 1956.

29. Mathupala SP, Ko YH and Pedersen PL: Hexokinase-2 bound to mitochondria: Cancer's stygian link to the 'Warburg effect' and a pivotal target for effective therapy. Semin Cancer Biol 19: 17-24, 2009.

30. Yoshino H, Enokida H, Itesako T, et al: Tumor-suppressive microRNA-143/145 cluster targets hexokinase-2 in renal cell carcinoma. Cancer Sci 104: 1567-1574, 2013. 